\title{
The Reliability of Ultrasound Diagnosis in Differentiating Malignant from Benign Thyroid Nodules Using TI-RADS Selection Followed by FNA
}

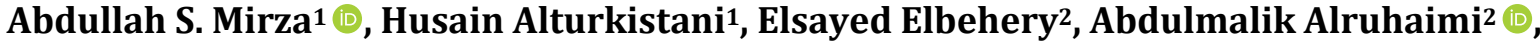 \\ Ahmed A. Mirza2 ${ }^{(1)}$, Syed 0. Ahsan² (D), Turki H. Alharbi2 ${ }^{(1)}$ \\ ${ }^{1}$ Radiology and Diagnostic Imaging Department, King Saud University Medical City, Riyadh, Saudi Arabia \\ ${ }^{2}$ Department of Medical Laboratory Science, King Abdulaziz University, Jeddah, Saudi Arabia \\ Email: Mirza.as@hotmail.com, Halturkistani@ksu.edu.sa, Elsayedabbas@yahoo.com, abdulmalikalruhaimi@gmail.com, \\ Amirza1@kau.edu.sa,drobaid_rad@yahoo.com, Turki14071017@gmail.com
}

How to cite this paper: Mirza, A.S., Alturkistani, H., Elbehery, E., Alruhaimi, A. Mirza, A.A., Ahsan, S.O. and Alharbi, T.H. (2021) The Reliability of Ultrasound Diagnosis in Differentiating Malignant from Benign Thyroid Nodules Using TI-RADS Selection Followed by FNA. Open Journal of Radiology, 11, 115-125.

https://doi.org/10.4236/ojrad.2021.113011

Received: June 24, 2021

Accepted: September 21, 2021

Published: September 24, 2021

Copyright $\odot 2021$ by author(s) and Scientific Research Publishing Inc. This work is licensed under the Creative Commons Attribution International License (CC BY 4.0).

http://creativecommons.org/licenses/by/4.0/ (c) (i) Open Access

\begin{abstract}
Context: Diagnostic imaging has increased the rate of thyroid nodules detection and improved utilization of fine-needle aspiration (FNA). Objective: This study aims to demonstrate the effects of the most reliable non-invasive technique on thyroid nodules. Methods: Between 2016 and 2020, 190 patients with 214 nodules visiting King Khalid University Hospital were randomly selected and retrospectively reviewed. Following the ACR TI-RADS recommendations for FNA and correlating cytology reports. Two expert radiologists with ultrasonographic imaging experience re-evaluated and reviewed the images. 88 nodules (41\%) in 79 patients were excluded because the nodule size was smaller than the FNA recommended size. Results: Following the ACR TI-RADS for FNA recommended selection, 27 nodules $(21.4 \%)$ out of the recommended 126 nodules were consistent with malignancy in cytology, with overall mean sensitivities, specificities, accuracies, precisions, and negative predictive values (NPV) of $96.4 \%, 40.7 \%, 48.7 \%, 28.4 \%$, and $98.6 \%$ respectively. The nodules were subdivided into the TI-RADS 3,4 , and 5 . Conclusion: In conclusion, ACR TI-RADS is feasible, reliable, and well structured, easily applicable in thyroid nodules reporting. ACR TI-RADS can eliminate many unnecessary FNAs, providing a decline in costs and complications. We recommend the ACR TI-RADS in our radiology department to eliminate reporting discrepancies and cut costs, thereby standardizing the reports, improving intra-user agreements, and improving overall patients' health care.
\end{abstract}




\section{Keywords}

Thyroid Cancer, Thyroid Nodules, Thyroid Ultrasound, Ultrasound, TIRADS

\section{Introduction}

Thyroid nodular disease is a common healthcare problem, prevalent among $20 \%$ - 50\% of the population [1]. However, thyroid malignancies are uncommon, only account for approximately $2 \%-7 \%$ of all thyroid nodules [1] [2] [3]. Thyroid cancer incidence has increased in the last few decades, without a significant change in the disease mortality. Diagnostic imaging has increased the rate of thyroid nodules detection and improved utilization of fine-needle aspiration (FNA) [4]. Ultrasound (US) guided FNA using the Bethesda [5] pathological evaluation system is the gold standard for thyroid nodules evaluation because of its cost-effectiveness and accurate detection rate [6]-[11]. Early assessment of thyroid nodules using the US has become the most valuable noninvasive diagnostic tool [12]. American College of Radiology (ACR) provides an easy-to-apply method for radiologists and physicians for proper management and increasing consistency across ultrasound practices. Thyroid Imaging Reporting and Data System (TI-RADS) [13], using the classification system, offers reliable, feasible, practical, and non-invasiveness techniques to eliminate $26 \%-53 \%$ of FNA cases [14] [15]. The aim of this study is to determine if using Ultrasound using TI-RADS selection can differentiate malignant from benign thyroid nodules confirmed by FNA. This will eliminate unnecessary invasive procedures for diagnostic purpose only in the management of thyroid nodules, thus avoiding unnecessary patients' morbidities, reflecting positively over the net cost.

\section{Methods and Materials}

\subsection{Patients}

Using the ACR TI-RADS, we randomly selected and retrospectively evaluated nodules in 190 patients visiting King Khalid University Hospital (KKUH) from 2016 to 2020, for thyroid US and FNA. A total of 126 nodules were evaluated in 111 patients; the outcome showed the absence of primary malignancy. Approximately 88 nodules in 79 patients were evaluated. Exclusion criteria were: 1) nodules without FNA recommendation according to the ACR TI-RADS, either because of size and/or grade (69 nodules). 2) Nodules with indefinite cytology, including any BETHESDA that scores more than 2 or 6 (19 nodules). No other exclusion criteria were applied. Table 1 provides a summary of all the evaluated nodules.

\subsection{Imaging and TI-RADS Scoring}

Two expert radiologists with ultrasonographic imaging experience re-evaluated and reviewed the images using the ACR TI-RADS protocols. To eliminate evalu- 
ation bias, both reviewers were blinded to the FNA cytology results before evaluation. The ACR TI-RADS was used to evaluate the ultrasonographic images, using five imaging evaluation criteria. For each feature, the calculated points were summed to determine the FNA recommendation and malignancy risk levels of each nodule, from TI-RADS 1 (lowest risk) to TI-RADS 5 (highest risk) [13]. Figure 1 provides the ACR-TI-RADS assessment categories. To confirm all the selected nodules, we validated the corresponding US-guided FNA procedure images and reviewed its correlated pathological report.

Table 1. Summary of the total evaluated nodules after applying ACR-TI-RADS and reviewing the corresponding cytology results.

\begin{tabular}{|c|c|c|c|c|}
\hline & $\begin{array}{c}\text { Number of } \\
\text { nodules }\end{array}$ & $\begin{array}{l}\text { Recommendatior } \\
\text { for FNA }\end{array}$ & $\begin{array}{l}\text { No recommendation } \\
\text { for FNA }\end{array}$ & $\begin{array}{c}\text { Nodules with conclusive } \\
\text { cytological results }\end{array}$ \\
\hline Included nodules & 126 & 126 & 0 & 126 \\
\hline Excluded nodules & 88 & 19 & 69 & 69 \\
\hline TOTAL & 214 & 145 & 69 & 195 \\
\hline
\end{tabular}

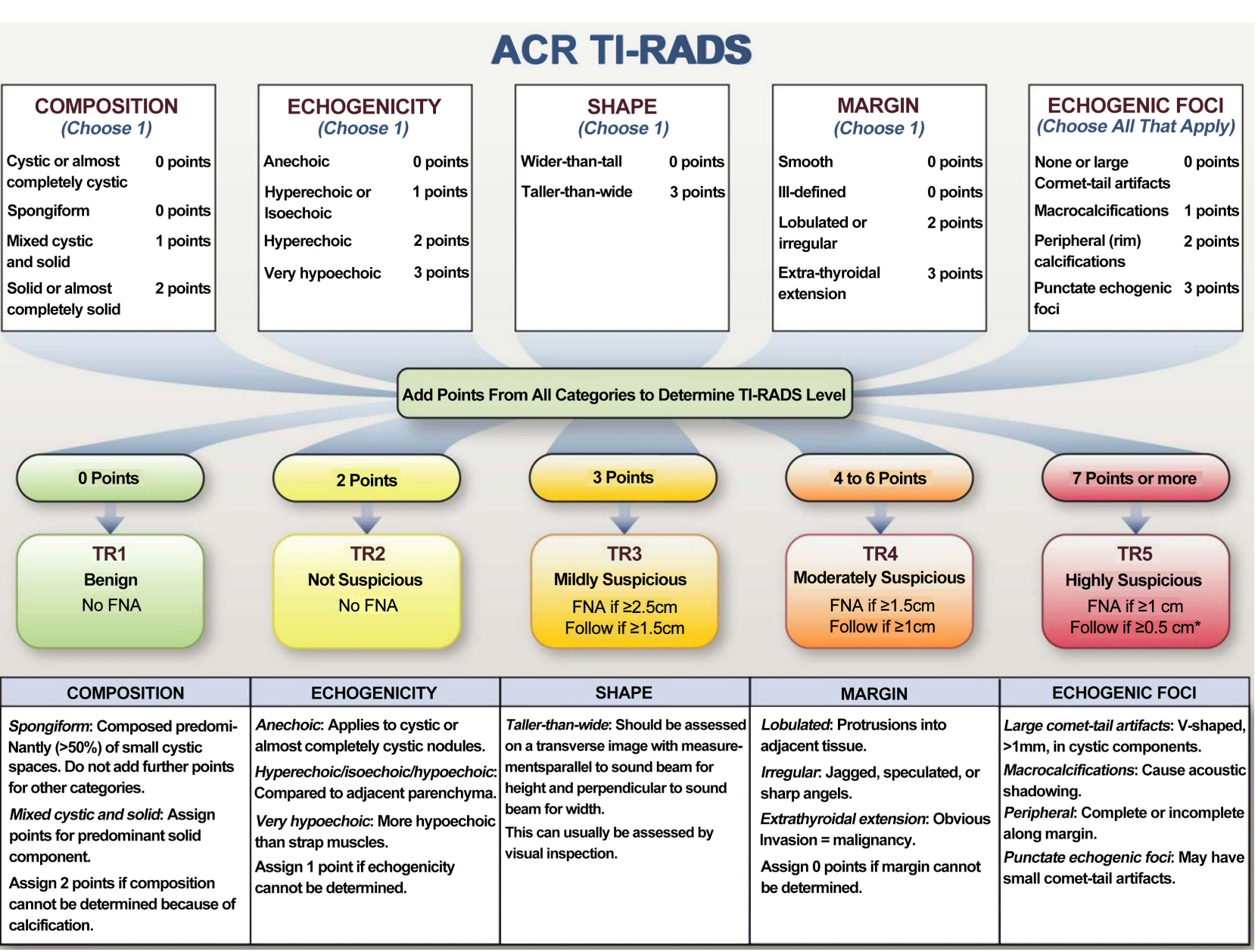

Figure 1. Assessment categories for thyroid nodules using ultrasound suggested by the ACR TI-RADS committee ${ }^{\star}$ Originally published in JACR at:

https://www.jacr.org/article/S1546-1440(17)30186-2/fulltext?_ga=2.188257452.525486094.1565030521-1858166925.1544129320). 


\subsection{FNA Technique and Cytology}

Well-trained and experienced interventional radiology consultants conducted all FNA procedures using local anesthesia. The US scan and guided biopsy images were reviewed to confirm the biopsied nodules. All the cytological samples were evaluated using the BETHESDA scoring system; with 1 unsatisfactory, 2 benign, 3 typical cells, 4 follicular neoplasms, 5 suspicious for malignancy, and 6 malignancy [5].

\section{Statistical Analysis}

\section{Study Population}

Between 2016 and 2020, 190 patients with 214 nodules visiting King Khalid University Hospital were randomly (non-consecutive) selected and retrospectively reviewed. Following the ACR TI-RADS recommendations for FNA and correlating cytology reports, 88 nodules (41\%) in 79 patients were excluded because the nodule size was smaller than the FNA recommended size, was of the improper initial nodule's measurements, incorrect TI-RADS category, or inconclusive result from cytology. ACR TI-RADS reliability evaluation was conducted on the rest of the 111 patients (95 Females and 16 Males) with 126 thyroid nodules (13 patients 9 females and 4 males with 2 nodules, and 1 female patient with 3 nodules). The male mean age is 49.4 (mean \pm SD $49.4 \pm 17$, maximum 69, and minimum 17); the female mean age is 47 (mean \pm SD $47 \pm 12.8$, maximum 74, and minimum 21). Table 2 summarizes the patient's demographic characteristics. The excluded nodules were 69 nodules (around 32\%) not included in the evaluation criteria. However, 19 nodules (about 9\%) were completely excluded owing to inconclusive pathology results. This study received approval from the Research Ethics Committee of Health Affairs in Riyadh, KSA (KSU-IRB 017E).

\section{Results}

Microsoft Excel was used to collect data. Afterward, we used SPSS version 22.0 for the analysis. The sensitivity, specificity, accuracy, precision, and negative predictive value (with a confidence interval of 95\%) were conducted to determine the ACR TI-RADS reliability and differentiate benign from malignant thyroid nodules. A p-value $<0.05$ was considered a significant level.

Table 2. Age and gender demographics of patients with FNA recommended by the ACR TI-RADS.

\begin{tabular}{cccc}
\hline Gender & Minimum age & Maximum age & Mean age (Std. Dev) \\
\hline Female & 21 & 74 & $47.03( \pm 12.76)$ \\
Male & 17 & 69 & $49.44( \pm 17.02)$ \\
All & 17 & 74 & $47.38( \pm 13.39)$ \\
\hline
\end{tabular}


A significantly moderate correlation and very strong association between TI-RADS and FNA results were observed using Spearman rho correlation ( $\mathrm{rs}(195)$ $=0.450, \mathrm{p}<0.001, \varphi$ Cramer $=0.520, \mathrm{p}<0.001) . \mathrm{A}$ Mann-Whitney U-test was used to evaluate the difference between the ages of patients with benign and malignant tumors. The result was significantly different $(\mathrm{Mdn}=5241.5,1724.5, \mathrm{p}<$ 0.05).

No other significant correlation was found between age, gender, site, and malignant or benign lesions.

Of the total 214 nodules, 19 nodules (18 patients) were excluded owing to their inconclusive cytology result (any BETHESDA score but 2 and 6). Thus, 195 nodules in 172 patients were properly evaluated using the ACR TI-RADS with an overall malignancy of $14.4 \%, 28$ nodules. Table 3 shows the percentage of malignancy in each category: $1.2 \%$ in TI-RADS 3 ( 1 out of 85 ), $14.1 \%$ in TI-RADS 4 (12 out of 85 ), and $60 \%$ in TI-RADS 5 (15 out of 25).

Following the ACR TI-RADS for FNA recommended selection, 27 nodules (21.4\%) out of the recommended 126 nodules were consistent with malignancy in cytology, with overall mean sensitivities, specificities, accuracies, precisions, and negative predictive values (NPV) of $96.4 \%, 40.7 \%, 48.7 \%, 28.4 \%$, and $98.6 \%$ respectively. The nodules were subdivided into the TI-RADS 3, 4, and 5. Table 4 summarizes the results of the ACR TI-RADS for malignancy detection, and Figure 2 plotted the ROC curve.

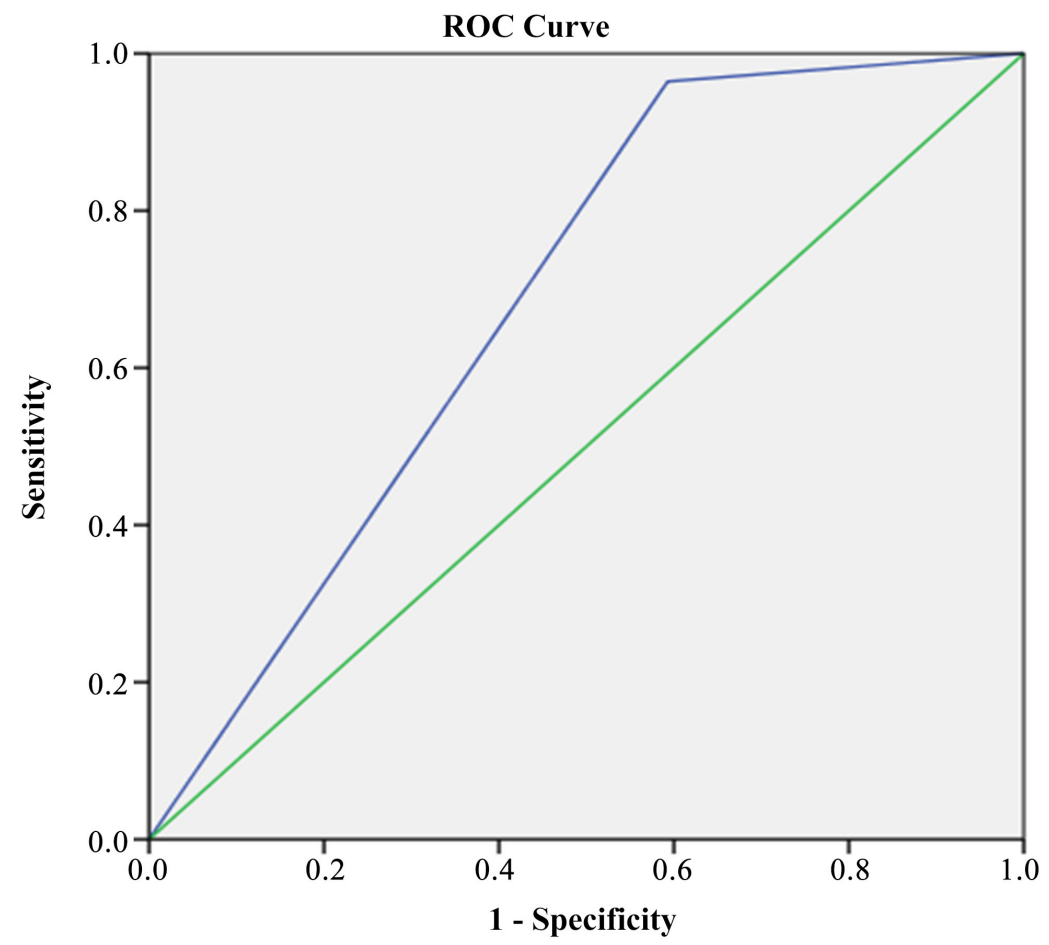

Diagonal segments are produced by ties.

Figure 2. ROC curve was to evaluate the specificity and selectivity of the TI-RADS system. 
Table 3. Total assessed ACR-TI-RADS nodules with conclusive cytology results.

\begin{tabular}{ccccc}
\hline TI-RADS category & Number of nodules & Benign nodules & Malignant nodules & Malignancy $\%$ \\
\hline TI-RADS 3 & 85 & 84 & 1 & $1.2 \%$ \\
TI-RADS 4 & 85 & 73 & 12 & $14 \%$ \\
TI*RADS 5 & 25 & 10 & 15 & $60 \%$ \\
TOTAL & 195 & 167 & 28 & $14.4 \%$ \\
\hline
\end{tabular}

Table 4. Thyroid nodule malignancy detection after the application of ACR-TI-RADS for nodule assessment, following FNA, recommended selection criteria.

\begin{tabular}{ccccc}
\hline & TI-RADS 3 & TI-RADS 4 & TI-RADS 5 & Total nodules \\
\hline Sensitivity & 100 & 91.7 & 100 & $96.4 \%$ \\
Specificity & 46.4 & 38.4 & 10 & $40.7 \%$ \\
PPV (precision) & 2.2 & 19.6 & 62.5 & $21.4 \%$ \\
NPV & 100 & 96.6 & 100 & $98.6 \%$ \\
Accuracy & 47.1 & 45.9 & 64 & $48.7 \%$ \\
\hline
\end{tabular}

TI-RADS 3 included 46 nodules (36.5\%): 1 malignant nodule (2.2\%) and 45 benign nodules (97.8\%), showing sensitivity, specificity, accuracy, and precision with an NPV of $100 \%, 46.4 \%, 47.1 \%, 2.2 \%$ and $100 \%$, respectively. TI-RADS 4 included 56 nodules (44.4\%): 11 malignant nodules (19.6\%) and 45 benign nodules (80.4\%), showing sensitivity, specificity, accuracy, precision with an NPV of $91.7 \%, 38.4 \%, 45.9 \%, 19.6 \%$, and $96.6 \%$, respectively. TI-RADS 5 included 24 nodules (19.1\%): 15 malignant nodules $(62.5 \%)$ and 9 benign nodules $(37.5 \%)$, showing sensitivity, specificity, accuracy, precision with NPV of $100 \%, 10 \%$, $64 \%, 62.5 \%$, and $100 \%$ respectively.

ROC curve was plotted to evaluate the specificity and selectivity of the TI-RADS system for the malignant thyroid nodules detection, AUC $=0.686$ (95\% CI, $0.598-0.773), \mathrm{p}<0.05$.

\section{Discussion}

While excluding thyroid malignancy using FNA is a gold standard for suspicious thyroid nodules evaluation, it is proven to be user-dependent based on the $20 \%$ non-diagnostic results [6] [7] [8] [9]. The procedure exposes patients to multiple sample collection, cytological evaluation, and more stress to the families. Thus, improving FNA diagnostic accuracy was mandatory. In addition, the ACR TI-RADS evaluation criteria are more effective than other classification methods because it improves the diagnostic accuracy, decreases unnecessary sampling, cut costs, and improves overall patient care [14] [15] [16]. Many radiologists are still reluctant to use the ACR TI-RADS evaluation tool because they are either unfamiliar or hesitant with it. Some are simply uncomfortable to change the "routine," thereby relying on personal experience. Thus, a simple and applicable selection criterion is critical to change the situation dramatically. 
In our retrospective study, recent and old ultrasonographic images completed between 2016 and 2020 were selected using a customized system filter that was based on patients' FNA history collected from their electronic files. Thus, cases might be subjected to selection and evaluation bias owing to the discrepancy in the included lesions versus excluded lesions (126 vs. 69) and evaluating cases of previous ACR TI-RADS classification (19 cases). For better accuracy and enhanced evaluation, we assigned well-trained and senior radiologists with over15 years of experience in diagnostic ultrasound to complete a blind review of the corresponding cytology reports. We reevaluated all the cases without further selection. Yet, cases in the lower ACR TI-RADS subcategories (TR-1 and TR-2) were minimal and could not be properly evaluated. However, the ACR and many recent studies concluded that the malignancy risk in TR-1 and TR-2 subcategories never exceeded $2 \%$ in the ACR partial analysis [13] and $0 \%$ of studies conducted by Warinthorn Phuttharak et al. [15] and Hoang et al. [17].

Our TI-RADS evaluation results were in agreement with the ACR TI-RADS paper that focused on malignancy risk stratification: Less than $5 \%$ in TR-3 (1.2\%), from $5 \%$ to $20 \%$ in TR-4 (14.1\%), and more than $20 \%$ in TR-5 (60\%) [13]. Figure 3 and Figure 4 provide examples of the evaluated nodules with corresponding cytology and histopathology. The classification system eliminated the FNA procedures in many non-recommended cases, which exceeded 32 $(32.4 \%)$ out of the initially included lesions. If well implemented, the procedure significantly reduces the costs.

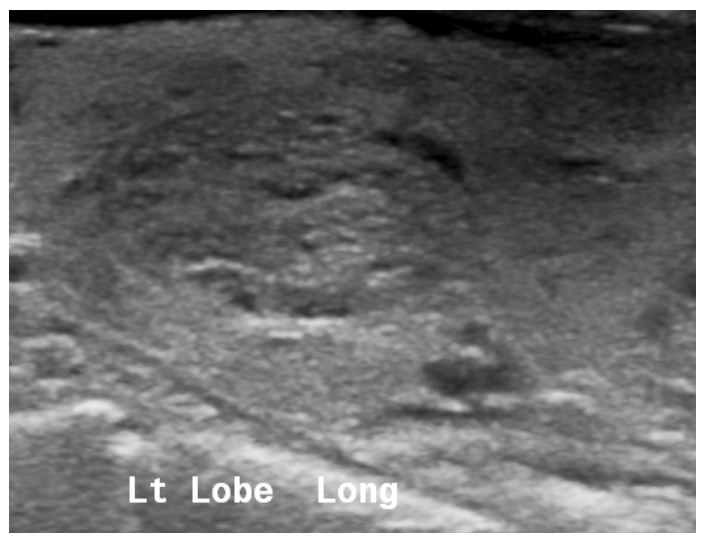

(a)

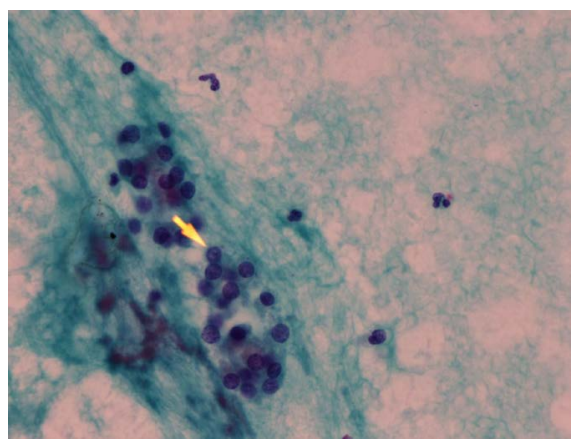

(b) 


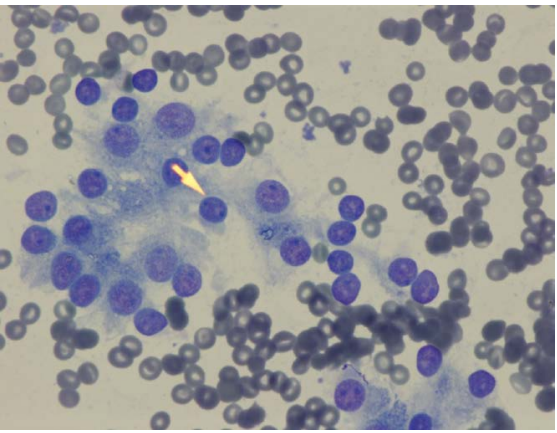

(c)

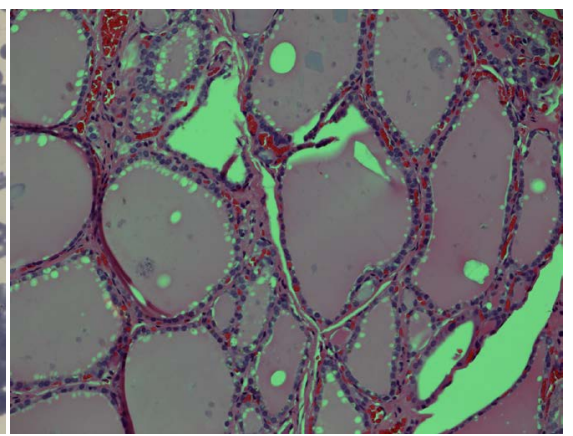

(d)

Figure 3. Ultrasound image of a TI-RADS 3 nodule assessed using the ACR TI-RADS with corresponding cytology slide. Cytology proven benign. (a) Case1-selected cropped US image. The left thyroid lobe contains a $2.6 \times 1.3 \mathrm{~cm}$ and defines isoechoic nodule, composed of predominantly solid with some cystic components (A non-suspicious nodule: TI-RADS 3 no FNA recommendation according to the ACR-TI-RADS). This case was cytology proven to be benign adenomatoid nodule; (b) Case1-Pap stain $\times 600$. Photomicrograph obtained from a thyroid FNA from the case number 2 showing benign thyroid follicular cells (arrowhead). This case was reported as Bethesda category 2 Pap stain $\times 600$; (c) Case1-DiffQuick stain $-\times 600$. Photomicrograph obtained from a thyroid FNA from case 1 showing benign thyroid follicular cells (arrowhead). This case was reported as Bethesda category 2 compatible with adenomatoid nodule. Diff-Quick stain $\times 60$; (d) Case1-HE stain $-\times 200$. Photomicrograph represents a histologic section, shows benign thyroid follicles, and confirms the previous cytology of Bethesda 2. H/E stain $\times 200$.

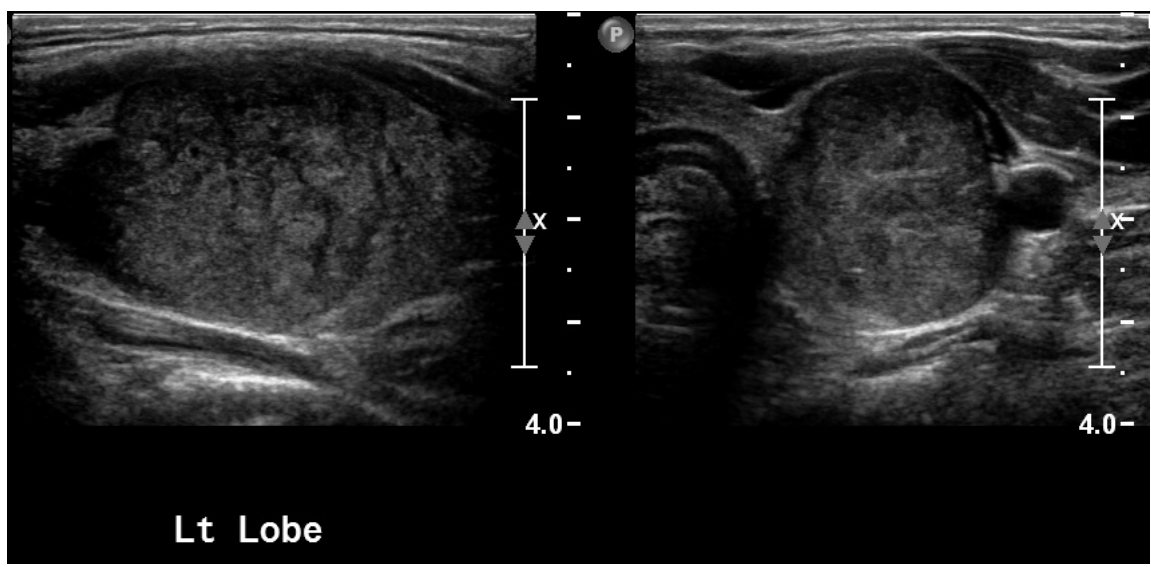

(a)

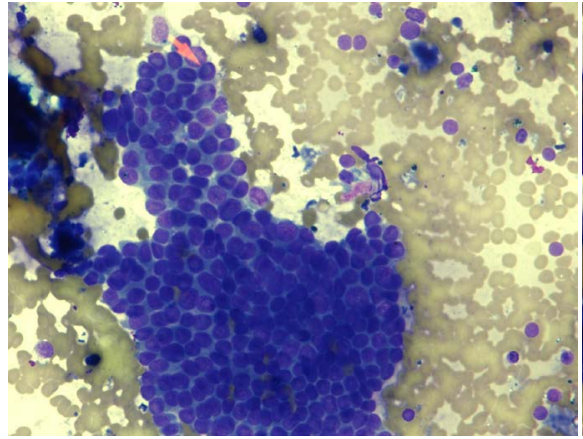

(b)

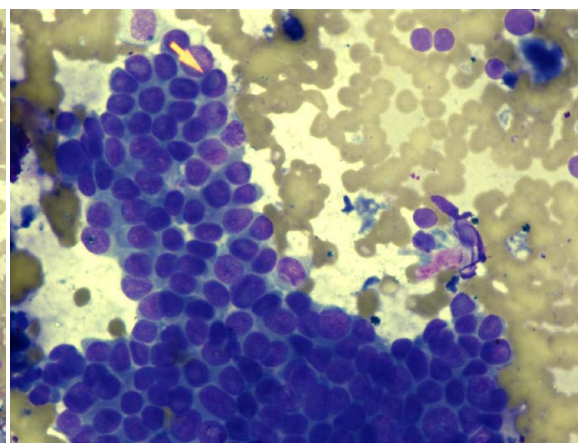

(c) 


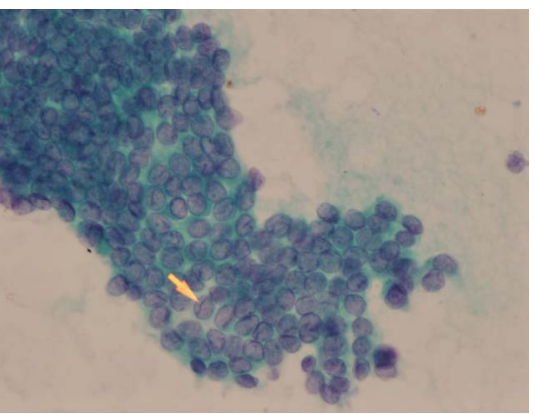

(d)

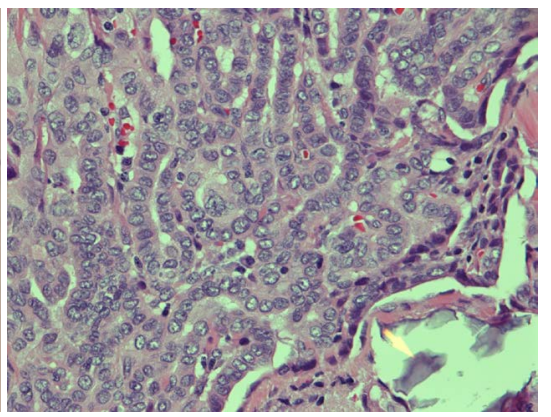

(e)

Figure 4. Ultrasound image of a TI-RADS 5 nodule assessment using the ACR TI-RADS with corresponding cytology and histopathology slides. Pathologically proven malignant. (a) Case 2-Selected cropped US images obtained in long and short axes. The left thyroid lobe shows a $2.7 \times 2 \mathrm{~cm}$ solid hypoechoic nodule, taller, and wider with echogenic focus of calcification (A highly suspicious nodule: TI-RADS 5 for FNA according to the ACR-TI-RADS). This case was cytology and histopathology proven to be malignant papillary thyroid carcinoma; (b) Case2-DiffQuick stain $-\times 400$. High power microscopic view of thyroid FNA obtained from case number 2 showing a typical cell showing nuclear overlapping and intranuclear inclusions (arrowhead). This case was reported as malignant papillary carcinoma, Bethesda 6. Diff-Quick cytology stain $\times 400$. (c) Case2-DiffQuick stain $-\times 600$. High power microscopic view of thyroid FNA obtained from case number 2 showing a typical cell, nuclear overlapping, and intranuclear inclusions (arrowhead). This case was reported as malignant papillary carcinoma, Bethesda 6. Diff-Quick cytology stain $\times 600$. (d) Case2-Pap stain $-\times 400$. Papanicolaou cytology stain preparation obtained from Case 2 showing clusters of a typical thyroid follicular cells with nuclear overlapping and distinct nuclear grooving (arrowhead). The features are compatible with papillary carcinoma. Papanicolaou stain $\times 600$; (e) Case 2 -HE stain $\times 400$. Histopathology section obtained from the thyroidectomy resection specimen of case number 2 confirmed classical papillary carcinoma of the thyroid gland. An area of calcification (arrowhead) is seen. $\mathrm{H} / \mathrm{E}$ stain $\times$.

From the application of ACR TI-RADS, we did not recommend FNA in 69 nodules (in 63 patients), with only 1 small TI-RADS 4 malignant lesions (approximately $0.5 \%$ of the total evaluated lesions and $1.5 \%$ of the excluded lesions). Although this nodule fails to receive an FNA recommendation used in the ACR TI-RADS due to its small size, this nodule is categorized as a TI-RADS 4 nodule, and it measures $1 \times 0.8 \mathrm{~cm}$ diameter. Hence, follow-up is not dismissed. Nonetheless, 62 patients went through a stressful procedure without proper imaging or medical justification, wasting medical and financial resources. In addition, the procedure is without standardized, clear diagnostic methods, and guidelines subjecting to many incorrect user-dependent judgmental discrepancies and resource misuse.

\section{Conclusion}

In conclusion, ACR TI-RADS is feasible, reliable, and well structured, easily applicable in thyroid nodules reporting. ACR TI-RADS can eliminate many unnecessary FNAs, providing a decline in costs and complications. We recommend the ACR TI-RADS in our radiology department to eliminate reporting discre- 
pancies and cut costs, thereby standardizing the reports, improving intra-user agreements, and improving overall patients' health care. Our future research topic is to find ancillary non-invasive modality to enhance the accuracy in differentiating malignant from benign thyroid nodules.

\section{Disclosure}

This work was not supported or funded by any drug or machine company.

\section{Acknowledgements}

We thank Professor Ammar C. Al Rikabi for his aid in acquiring and reporting cytology and histopathology images.

\section{Conflicts of Interest}

The authors declare no conflicts of interest regarding the publication of this paper.

\section{References}

[1] Pacini, F., Schlumberger, M., Dralle, H., Elisei, R., Smit, J.W. and Wiersinga, W. (2006) European Consensus for the Management of Patients with Differentiated Thyroid Carcinoma of the Follicular Epithelium. European Journal of Endocrinology, 154, 787-803. https://doi.org/10.1530/eje.1.02158

[2] Campennì, A., Giovanella, L., Siracusa, M., Stipo, M.E., Alibrandi, A., Cucinotta, M., Ruggeri, R.M. and Baldari, S. (2014) Is Malignant Nodule Topography an Additional Risk Factor for Metastatic Disease in Low-Risk Differentiated Thyroid Cancer? Thyroid, 24, 1607-1611. https://doi.org/10.1089/thy.2014.0217

[3] Welker, M.J. and Orlov, D. (2003) Thyroid Nodules. American Family Physician, 67, 559-566.

[4] Chen, A.Y., Jemal, A. and Ward, E.M. (2009) Increasing Incidence of Differentiated Thyroid Cancer in the United States, 1988-2005. Cancer. Interdisciplinary International Journal of the American Cancer Society, 115, 3801-3807. https://doi.org/10.1002/cncr.24416

[5] Cibas, E.S. and Ali, S.Z. (2009) The Bethesda System for Reporting Thyroid Cytopathology. Thyroid, 19, 1159-1165. https://doi.org/10.1089/thy.2009.0274

[6] Brito, J.P., Gionfriddo, M.R., Al Nofal, A., Boehmer, K.R., Leppin, A.L., Reading, C., Callstrom, M., Elraiyah, T.A., Prokop, L.J., Stan, M.N. and Murad, M.H. (2014) The Accuracy of Thyroid Nodule Ultrasound to Predict Thyroid Cancer: Systematic Review and Meta-Analysis. The Journal of Clinical Endocrinology \& Metabolism, 99, 1253-1263. https://doi.org/10.1210/jc.2013-2928

[7] Gharib, H., Papini, E., Paschke, R., Duick, D., Valcavi, R., Hegedüs, L. and Vitti, P. (2010) American Association of Clinical Endocrinologists, Associazione Medici Endocrinologi, and European Thyroid Association Medical Guidelines for Clinical Practice for the Diagnosis and Management of Thyroid Nodules. Endocrine Practice, 16, 1-43. https://doi.org/10.4158/10024.GL

[8] Haugen, B.R., Alexander, E.K., Bible, K.C., Doherty, G.M., Mandel, S.J., Nikiforov, Y.E., Pacini, F. Randolph, G.W., Sawka, A.M., Schlumberger, M. and Schuff, K.G. (2016) American Thyroid Association Management Guidelines for Adult Patients 
with Thyroid Nodules and Differentiated Thyroid Cancer: The American Thyroid Association Guidelines Task Force on Thyroid Nodules and Differentiated Thyroid Cancer. Thyroid, 26, 1-33. https://doi.org/10.1089/thy.2015.0020

[9] Baloch, Z.W., Cibas, E.S., Clark, D.P., Layfield, L.J., Ljung, B.M., Pitman, M.B. and Abati, A. (2008) The National Cancer Institute Thyroid Fine Needle Aspiration State of the Science Conference: A Summation. Cytojournal, 5, 6.

https://doi.org/10.1186/1742-6413-5-6

[10] Perros, P., Boelaert, K., Colley, S., Evans, C., Evans, R.M., Gerrard, B.A.G., Gilbert, J., Harrison, B., Johnson, S.J., Giles, T.E. and Moss, L. (2014) Guidelines for the Management of Thyroid Cancer. Clinical Endocrinology, 81, 1-222. https://doi.org/10.1111/cen.12515

[11] Cooper, D.S., Doherty, G.M., Haugen, B.R., Kloos, R.T., Lee, S.L., Mandel, S.J., Mazzaferri, E.L., McIver, B., Sherman, S.I. and Tuttle, R.M. (2006) Management Guidelines for Patients with Thyroid Nodules and Differentiated Thyroid Cancer: The American Thyroid Association Guidelines Taskforce. Thyroid, 16, 109-142. https://doi.org/10.1089/thy.2006.16.109

[12] Chaudhary, V. and Bano, S. (2013) Thyroid Ultrasound. Indian Journal of Endocrinology and Metabolism, 17, 219. https://doi.org/10.4103/2230-8210.109667

[13] Tessler, F.N., Middleton, W.D., Grant, E.G., Hoang, J.K., Berland, L.L., Teefey, S.A., Cronan, J.J., Beland, M.D., Desser, T.S., Frates, M.C., Hammers, L.W., Hamper, U.M., Langer, J.E., Reading, C.C., Scoutt, L.M. and Stavros, A.T. (2017) ACR Thyroid Imaging, Reporting and Data System (TI-RADS): White Paper of the ACR TI-RADS Committee. Journal of the American College of Radiology, 14, 587-595. https://doi.org/10.1016/j.jacr.2017.01.046

[14] Grani, G., Lamartina, L., Ascoli, V., Bosco, D., Biffoni, M., Giacomelli, L., Maranghi, M., Falcone, R., Ramundo, V., Cantisani, V., Filetti, S. and Durante, S. (2019) Reducing the Number of Unnecessary Thyroid Biopsies While Improving Diagnostic Accuracy: Toward the "Right" TI-RADS. The Journal of Clinical Endocrinology \& Metabolism, 104, 95-102. https://doi.org/10.1210/jc.2018-01674

[15] Jabar, A.S.S., Prakashini, K. and Jasbon, A. (2019) Diagnostic Reliability of the Thyroid Imaging Reporting and Data System (TI-RADS) in Routine Practice. Polish Journal of Radiology, 84, e274. https://doi.org/10.5114/pjr.2019.86823

[16] Phuttharak, W., et al. (2019) Interrater Reliability of Various Thyroid Imaging Reporting and Data System (TI-RADS) Classifications for Differentiating Benign from Malignant Thyroid Nodules. Asian Pacific Journal of Cancer Prevention, 20, 1283-1288. https://doi.org/10.31557/APJCP.2019.20.4.1283

[17] Hoang, J.K., Middleton, W.D., Farjat, A.E., Langer, J.E., Reading, C.C., Teefey, S.A., Hertzberg, B.S., et al. (2018) Reduction in Thyroid Nodule Biopsies and Improved Accuracy with American College of Radiology Thyroid Imaging Reporting and Data System. Radiology, 287, 185-193. https://doi.org/10.1148/radiol.2018172572 